NOTICE: This is the author's version of a work that was accepted for publication in Research in Autism Spectrum Disorders. Changes resulting from the publishing process, such as peer review, editing, corrections, structural formatting and other quality control mechanisms may not be reflected in this document. Changes may have been made to this work since it was submitted for publication. A definitive version was subsequently published in Research in Autism Spectrum Disorders, Volume 8, Issue 5, May 2014, Pages 559-569. http://doi.org/10.1016/j.rasd.2014.01.014 


\section{Visual search strategies during facial recognition in children with ASD}

Matthew A Albrecht ${ }^{1}$, Jonathan K Foster ${ }^{1,2}$, Annette Joosten ${ }^{3}$, Marita Falkmer ${ }^{3,4}$, Julia Tang ${ }^{3}$, Denise Leung $^{3}$, Anna Ordqvist ${ }^{3}$, Torbjorn Falkmer ${ }^{3,5,6,7, *}$

1. School of Psychology and Speech Pathology, Curtin University, Perth, Western Australia, Australia

2. Neurosciences Unit, Health Department of WA, Perth, Western Australia, Australia

3. School of Occupational Therapy \& Social Work, CHIRI, Curtin University, Perth, WA, Australia

4. School of Education and Communication, CHILD programme, Institute of Disability Research, Jönköping University, Sweden

5. Department for Rehabilitation, School of Health Sciences, Jönköping University, Jönköping Sweden

6. Rehabilitation Medicine, Department of Medicine and Health Sciences (IMH), Faculty of Health Sciences, Linköping University \& Pain and Rehabilitation Centre, Linköping, Sweden

7. School of Occupational Therapy, La Trobe University, Melbourne, VIC, Australia

* Corresponding author: Professor Torbjörn Falkmer, Senior Research Fellow at the School of Occupational Therapy and Social Work, Curtin Health Innovation Research Institute (CHIRI), Faculty of Health Sciences, Curtin University of Technology, GPO Box U1987, Perth, Western Australia, 6845 Australia. Tel.: +61 89266 9051; fax: +61 89266 3636. E-mail:

T.Falkmer@curtin.edu.au - this email address can be published

Word Count

Abstract: 169

Main text: 3565

No Figures: 6

No Tables: 0 


\begin{abstract}
Facial recognition is a complex skill necessary for successful human interpersonal and social interactions. Given that the most prevalent disorder of social interaction is autism spectrum disorder (ASD), a number of studies have investigated and found impaired facial recognition abilities in people with ASD. Further, this impairment may be critically involved in mediating the deficits in interpersonal and social interactions in people with ASD. We sought to address the question of whether face processing is impaired in children with ASD in the current study. While there were a number of differences in visual search behaviours between the 19 children with ASD and the 15 controls, this did not manifest in deficits in facial recognition accuracy. In addition, there were notable differences with respect to eye fixation behaviours and recognition accuracy in this study compared to the findings in a previous similar study conducted in adults with ASD. These differences suggest a performance enhancing developmental trajectory in facial processing in controls that may not be present in individuals with ASD.
\end{abstract}

Keywords: Face recognition; eye-tracking; developmental; visual search 
Albrecht et al. 3

\section{Introduction}

Processing of faces is very important for human interpersonal and social interactions (Ellis, 1990), with the ability to recognise the identity of faces representing a key social attribute (BarHaim, Shulman, Lamy, \& Reuveni, 2006). Facial perception and subsequent recognition is a highly developed visual perceptual skill that is dependent on specialised neural circuitry (Haxby, Hoffman, \& Gobbini, 2002; McKone, Kanwisher, \& Duchaine, 2007; Pitcher, Charles, Devlin, Walsh, \& Duchaine, 2009; Tsao, Freiwald, Tootell, \& Livingstone, 2006; Tsao, Moeller, \& Freiwald, 2008) and has also been shown to be partly heritable (Wilmer et al., 2010). Effective/efficient facial recognition greatly facilitates interpersonal interactions via triggering memories of relevant personspecific social information, thereby enabling the prediction of future behaviour learnt from that individual's past behaviour.

Successful facial recognition requires effective strategies for extraction and encoding of invariant (to that person) facial structural features (O’Toole, 2011). Facial recognition accuracy has repeatedly shown to be impaired in individuals with ASD compared with controls (Weigelt, Koldewyn, \& Kanwisher, 2012). This impairment in identity recognition has been suggested to play a role in the social deficits present in individuals with ASD (Dawson, Webb, \& McPartland, 2005; Schultz, 2005). Facial recognition difficulties in ASD may be due to sub-optimal strategies for facial processing and recognition (Sasson, 2006). When participants with ASD and controls view faces during facial recognition tasks, both groups spend more time fixating on the eyes (or eye regions) compared to other regions of the face (Falkmer, Larsson, Bjällmark, \& Falkmer, 2010; Hernandez et al., 2009; Sterling et al., 2008). The eyes, in combination with the nose and mouth regions, form the core components of the "face information triangle" and receive the most attention during face perception (Yarbus, Haigh, \& Rigss, 1967). Relative to controls, individuals with ASD have been shown to spend less time fixating on the eyes (Hernandez et al., 2009; Sterling et al., 2008) and other core components of the face information triangle (Chawarska \& Shic, 2009; 
Albrecht et al. 4

Wilson, Palermo, \& Brock, 2012).

In contrast, Chawarska \& Shic (2009) reported conflicting evidence showing that attention to the eyes, nose and mouth declined from 2 to 4 years of age in children with ASD but both 2 and 4 year olds with ASD spent less time looking at the mouth compared with controls. However, there were no differences in the duration spent looking at the eyes. Furthermore, Snow et al. (2011) found that adolescents with ASD made less fixations outside of the face information triangle compared with controls. Nevertheless, the combined evidence in adults and children with ASD supports the notion that individuals with ASD manifest a somewhat different style of face processing (Sasson, 2006).

Part of the inconsistency between studies may be related to differences in the age of participants across studies and the differences in the use of configural processing versus feature processing of facial stimuli (Maurer, Grand, \& Mondloch, 2002). It has been demonstrated that younger children tend to use feature processing (i.e., prominent individual facial features) more for facial recognition, in contrast to older children who begin to use configural processing strategies (i.e., information is derived from the relations between facial features) to categorise faces (Jemel, Mottron, \& Dawson, 2006; Karatekin, 2007; Maurer et al., 2002). Individuals with ASD have been shown to possess a local versus global processing bias (Happé, 1999; Happé \& Frith, 2006; Shah \& Frith, 1983), which would suggest a bias for featural processing or a developmental delay in configural processing in individuals with ASD (Karatekin, 2007).

One method of examining whether featural or configural processing is being used during facial recognition tasks is to manipulate prominent features of facial stimuli (e.g., by segmenting a photograph of a face into pieces of facial puzzles; Falkmer et al., 2010). We previously demonstrated that adults with ASD displayed greater difficulties in facial recognition during a task where training stimuli were segmented into a number of pieces (Falkmer et al., 2010). In particular, adults with ASD performed worse when the training stimuli were segmented through the eyes 
Albrecht et al. 5

(effectively distorting the information that was available to the participant from the eyes) compared with controls, suggesting that adults with ASD relied more upon intact information from eye regions for facial recognition. In addition to the recognition accuracy results, we also found that individuals with ASD made shorter fixations (on average) to the eyes and mouth when using puzzle piece stimuli. By contrast, during the response recognition phase which used whole pictures, the adults with ASD made fixations of longer duration to the eyes and other areas of the face compared with controls (Falkmer et al., 2010).

In the current study we used this approach to determine whether children with ASD processed faces differently compared with typically developing controls. Substantial differences in facial information processing for facial recognition between children with ASD and adults with ASD are likely, especially considering age-related differences in face processing in the general population (Jemel et al., 2006; Karatekin, 2007; Maurer et al., 2002). We identified a homogeneous group of children with Asperger syndrome without comorbidity or intellectual disabilities and used sophisticated eye tracking equipment to investigate whether there are differences in the way people with ASD qualitatively (how facial identity is remembered) and/or quantitatively (how well facial identity is discriminated) (Weigelt et al., 2012) process facial information, compared with controls. Building on previous findings reported by Falkmer et al. (2010), we focused on the number, duration and pattern of eye fixations. We anticipated that children with ASD would have greater difficulties in identifying faces compared with controls, specifically when salient information in the eye region is distorted. We were also interested in determining whether children with ASD would display similar visual search behaviour to that documented previously in Falkmer et al. (2010) on the same face recognition task. 
Albrecht et al. 6

\section{Materials and Methods}

\section{Research participants}

Thirty-one children with HFA/AS and thirty-three control children were recruited to take part in the study. Twelve children with HFA/AS and 17 control children were excluded due to recording difficulties or participant attrition (the face recognition task was the last in a battery of tests administered, reported elsewhere; Falkmer, Bjällmark, Larsson, \& Falkmer, 2011). Nineteen children (4 Female) with HFA/AS and 15 control children (2 Female) completed the study. All children were aged between 8.6 and 12.7 years old $($ mean $[\mathrm{SD}]$ age HFA/AS = 10.6 [1.2]; controls = 10.8 [1.4]). Participants were recruited through the Telethon Institute for Child Health Research, local primary schools, personal contacts, and local radio and newspaper advertisements in the Perth metropolitan region in Western Australia. The inclusion criteria for the study specified children with High Functioning Autism (HFA)/Asperger syndrome (AS) who were able to read and understand written and verbal instructions in English. Medical records were sighted to confirm diagnosis. All children with HFA/AS were diagnosed by qualified professionals in independent medical clinics Children with comorbid cognitive impairments or developmental disorders were excluded.

This study was approved by the Human Research Ethical Committee of Curtin University (approval numbers OTSW-03-2011 and OTSW-10-2011). Informed assent from the participants and written informed consent from their parents/guardians were obtained prior to commencing the trial. Participants were given two cinema tickets as tokens of appreciation for participating in the study.

\section{Eye Tracking}

The set-up of the head-mounted Arrington ViewPointTM eye tracker used was the same as in a previous study (see Leung, Ordqvist, Falkmer, Parsons, \& Falkmer, 2013). The eye tracker was used to record eye movements of one eye (usually the right). However, if the visual tracking screening showed that the left eye was the better option, the eye tracker was set to record the 
Albrecht et al. 7

movements of the left eye. An Arrington Ultra Precision Head PositionerTM was used to stabilise the participants' head to minimise systematic error. Participants were permitted to wear their glasses when necessary. A 16-point calibration conducted in the beginning of the trial. In order to maintain calibration, the participants were asked to fixate on a black dot displayed on a white screen in between stimuli presentation. The analysis of eye tracking measurements was done according to a centroid mode algorithm (Falkmer, Dahlman, Dukic, BjäLlmark, \& Larsson, 2008).

Stimuli

[Place Figure 1 about here]

The term face recognition has been used in the literature to refer to a range of somewhat distinct abilities, with the methods used frequently differing between studies (Jemel et al., 2006). For example, Weigelt et al. (2012) present a review of face identity recognition in ASD, encompassing reference to tasks which have been used to measure face memory, face perception and the face inversion effect. We here focused on the ability to match faces from the presentation of only partial information (see Falkmer et al., 2010). Specifically, in the current study, we presented segmented faces and asked participants to perform a matching task with simultaneously presented whole faces (procedures of the presentation of the trail is shown in Figure 1). The segmented face matched only one of the other presented whole faces and the eyes were either presented as a whole or bisected, as shown in Figure 1 and 2. The different presentation of the eyes provides information on whether the eyes are important in face identification. The participants viewed 12 face identification trials with equal number of males and females. Face recognition accuracy is determined by correct identification of the non-identical segmented photo to one of the three whole faces presented. There was no time limit given to respond. Participants were not informed on whether the identification 
Albrecht et al. 8

was correct or not. Before the trial, participants were given explanation the procedures and a test trial was conducted.

Analyses of measurements

[Place Figure 2 about here]

As in the previous study by Falkmer et al. (2010) the analysis of the puzzle pieced photos and the response photos were divided into three different classifications, the ‘eye', 'mouth’ or 'other' (parts of the face) depending on the most prominent features of the face. Figure 2 shows an example of how these areas are classified.

Statistical analyses.

Data were analysed using Bayesian repeated measures models (similar to repeated measures ANOVA models) in R version 3.0.1 (R Development Core Team, 2012) using the “rjags” package to link with the Gibbs sampler "JAGS” (Plummer, 2003, 2011). The number of fixations on each of the areas of interest were analysed by incorporating a logistic link function for the binomial distribution into the repeated measures model. Each of the areas of interest was analysed separately by classifying each fixation on a specified area as a "Yes" and fixations on other areas as a "No". The posterior of the binomial repeated measures model yielded a distribution of credible odds ratios for the relative likelihood of fixating on a particular area of interest (compared to all other areas). From the posterior, the means $+95 \%$ highest density intervals were extracted.

For the fixation durations, a similar Bayesian repeated measure model was used with the exception that a $t$-distribution was used to model the error distribution, with the degrees of freedom parameter estimated from the data. The $t$-distribution was used over the normal distribution because it provides more robust estimates of central tendencies compared to those obtained from Gaussian 
Albrecht et al. 9

distributions (Kruschke, 2013).

The analysis scripts were adapted from the split-plot scripts by (Kruschke, 2011). The factors included in each model were group (2 levels - ASD vs control) by eyes split (2 levels - eyes split vs eyes whole). Puzzled faces were analysed in separate models to the whole faces. Priors for each of the parameters of interest (main effect of group, main effect of eyes split and the interaction between eyes split and group) and the participant level 'random' effects were described by a series of normal distributions centred on 0 with a standard deviation (SD) estimated from a half-Cauchy distribution (Gelman \& Hill, 2007). The scale parameter for all half-Cauchy SD priors was estimated from the data and the prior was described by a uniform distribution between 0 and 1,000. The prior on the normality parameter (degrees of freedom parameter for the $t$-distribution) for the durations model was described by an exponential distribution centred on 30 .

In total, 10,000 adaptation steps were used to tune the samplers, 20,000 burn-in steps were discarded before taking 200,000 samples from the posterior spread across three separate chains. All chains showed good convergence of the final parameters, and there was sufficiently low autocorrelation in the chains yielding a high number of effective samples. The $95 \%$ highest density intervals (HDI) of the posterior distributions were used to describe the credible interval for each of the parameter estimates and contrasts. 
Albrecht et al. 10

\section{Results}

Response Accuracy

There was no difference in overall response accuracy between children with ASD and controls. Controls obtained 66\% and 70\% response accuracy when the eyes were whole and bisected, respectively, while children with ASD obtained 62\% and 66\% accuracy (OR of more correct responses in the ASD group $=0.90,95 \%$ HDI $=0.62,1.25$ ). There was no discernible influence of eyes bisected or whole on response accuracy (OR of more correct responses for bisected eyes $0.89,95 \% \mathrm{HDI}=0.61,1.23$ ) and there were no interactions between diagnostic status and eye bisection on correct responses (eyes whole ASD - controls OR contrast $=0.90,95 \%$ HDI $=$ 0.57, 1.35; eyes bisected ASD - controls contrast $=0.89,95 \% \mathrm{HDI}=0.56,1.34$ ).

\section{Total Fixations}

The combined sample made a total of 15,020 fixations. The group with HFA/AS made 8,464 fixations while the controls made 6,556 fixations.

\section{Fixations - Puzzle Pieces}

Figure 3 presents the odds ratios and contrasts (ASD-controls) for fixations on the puzzle pieces. Children with ASD were slightly more likely to make fixations on the eyes and the mouth pieces compared to all other fixation locations. There appeared to be an interaction between diagnostic status and eye bisection when analysing eye fixations relative to mouth fixations. However, the credible interval for the interaction contrast contained 0 (interaction OR contrast $=$ $1.23,95 \% \mathrm{HDI}=0.99,1.52$ ) and there was no strong $a$ priori reason for such an interaction. Children with ASD were also much more likely to make fixations only on a puzzle piece rather than elsewhere. 
Albrecht et al. 11

\section{Fixations - Response Slides}

Figure 4 presents the odds ratios and contrasts (ASD-controls) for fixations on the response slides. In contrast to the puzzle pieces, children with ASD were less likely to make fixations on the eyes or the mouth. However, they were not less likely to fixate on the eyes relative to the mouth during the presentation of the response slides (contrast ASD - controls $=1.19,95 \%$ HDI $=0.98$, 1.45). In addition, children with ASD were more likely to fixate on other regions of the response slides that were not the eyes or the mouth compared with controls.

\section{Durations - Puzzle Pieces}

Figure 5 presents the mean fixation durations for the fixations to the puzzle pieces. There were no differences in mean fixation durations between children with ASD and controls.

\section{Durations - Response Slides}

Figure 6 presents the mean fixation durations for the fixations to the response slides. Children with ASD had a longer mean fixation duration on the eye sections for both bisected eyes and whole eyes compared with controls. There were no other differences in mean fixation durations between children with ASD and controls. 


\section{Discussion}

In the present study we were interested in determining whether faces would be processed differentially in children with ASD compared with controls. We identified a homogeneous group of children with Asperger syndrome without comorbidity such as intellectual disability, and used sophisticated eye tracking equipment to investigate whether children with ASD would process face information in a qualitatively and/or quantitatively different manner. Building on previous findings reported by Falkmer et al., (2010), we focused on the number, duration and pattern of eye fixations in order to evaluate both accuracy and speed

Predicated on the previous findings in adults with ASD obtained by Falkmer et al., (2010), we anticipated that children with Asperger syndrome would have greater difficulties in identifying faces compared with controls, specifically in relation to salient information presented in the eye region. We were also interested in determining whether children with Asperger syndrome would differentially utilise individual perceptual features rather than configural processing in processing faces, when compared with controls.

Our findings indicated that children with ASD were slightly more likely to fixate on the eyes and mouth during the puzzle phase but were less likely to fixate on eyes and mouth during the response task when the pictures were of whole faces. In addition, children with ASD made fixations of longer duration to the eyes and mouth during the face recognition slides (despite making fewer fixations) which was consistent with adults with ASD (Falkmer, 2010). However, these differences in visual search behaviour in the ASD group did not correspond with strong evidence for deficits in facial recognition accuracy. In particular, there were no notable diagnosis by eye bisection interactions for recognition accuracy.

\section{Facial recognition accuracy}

The impairment in face recognition experienced by people with ASD may be critical in 
understanding deficits in interpersonal and social interactions in autism (Jemel et al., 2006; von Hofsten, Uhlig, Adell, \& Kochukhova, 2009). However, when directly compared with adults with ASD (Falkmer et al., 2010), the present study did not find strong evidence for the same deficits in facial recognition accuracy in children with ASD. When puzzle pieces were segmented through the eyes, Falkmer et al., (2010) found reductions in face recognition accuracy (63\% in ASD vs 83\% in controls). While the direction of the deficit was similar in the present study using children, the magnitude of the difference was much smaller (66\% in ASD vs $70 \%$ in controls). The most obvious difference between the two studies is the age of the participants with children aged $8.6-12.7$ years compared to adult participants in the first study (Falkmer et al., 2010). Across studies, the control adults were more accurate overall than the control children ( $80 \%$ accuracy in adults versus $68 \%$ accuracy in children), while the adults with ASD performed at a comparable rate to the children with ASD in the present study ( 67\% accuracy in adults versus $64 \%$ accuracy in children). This suggests that facial recognition abilities continue to improve throughout adolescence and early childhood in controls, but not in people with ASD.

\section{Visual search behaviour: Fixations}

In comparing visual search behaviour between Falkmer et al. (2010) and the present study, adults with ASD made less fixations to the eyes than the controls at the expense of fixations to other areas when viewing the puzzled face. By contrast, children with ASD in the present study were more likely to fixate on the eye and mouth pieces compared to control children. An increase in fixations on eye and mouth pieces and a reduction in non-task relevant areas in the present study may be another possible explanation for the lack substantial recognition accuracy differences in children with ASD. The eye and mouth regions provide key pieces of information for face recognition and increased attention towards these regions (and reduced attention towards non-task relevant areas) is likely to improve facial encoding, yielding better recognition performance 
(Falkmer et al., 2010; Weigelt et al., 2012; Wilson et al., 2012). When participants viewed the response slides, the children with ASD in the present study made less fixations to the eye and mouth regions and more fixations to other regions, mirroring the findings in adults with ASD reported in Falkmer et al. (2010). This result contrasts with the findings obtained during the puzzle piece viewing/encoding. Falkmer et al. (2010) suggested that the reduction in eyes and mouth fixations in their adults with ASD reflected a poorer structured visual search strategy in the ASD participants neglecting more often the 'face information triangle' (Yarbus et al., 1967).

\section{Visual search behaviour: Durations}

For the adults with ASD (Falkmer et al., 2010) the average fixation duration for the eye and mouth puzzle pieces was shorter compared with controls, while in the present study there was no credible difference between children with ASD and controls. The difference between studies may suggest a developmental aspect associated with piecing together information from puzzled faces. For example, control adults may be able to quickly (comparatively to people with ASD) categorise a piece as a necessary part of the "face information triangle" and spend longer fixating on these pieces to extract information for encoding. However, control children may also still be developing this skill, resulting in little behavioural difference between children with ASD and control children. By contrast, children with ASD had a longer mean fixation duration on the eye regions when viewing response slides consistent with Falkmer et al. (2010). Again, Falkmer et al. (2010) suggested that the longer mean fixation time in ASDs reflected difficulties in the ability to use the information embedded within the "facial information triangle".

\section{Conclusions}

While there were a number of differences in visual search behaviour between children with ASD and controls, this did not manifest in deficits in facial recognition accuracy in the children with ASD. In addition, there were a number of differences between our previous study that recruited 
adults with ASD (Falkmer et al, 2010) and the present study in children. The most notable differences between the two age groups was on fixation behaviour to the puzzle pieces and on recognition accuracy. These differences suggest a performance enhancing developmental trajectory in facial processing in controls that may not present in individuals with ASD.

\section{Acknowledgements}

We would like to thank our participants and their families for taking part in our research. We would also like to acknowledge AIM Employment and Annete Wallerman from AUW-Konsult for additional data entering. 
Albrecht et al. 16

\section{References}

Bar-Haim, Y., Shulman, C., Lamy, D., \& Reuveni, A. (2006). Attention to eyes and mouth in highfunctioning children with autism. Journal of Autism and Developmental Disorders, 36(1), 131-137. doi:10.1007/s10803-005-0046-1

Chawarska, K., \& Shic, F. (2009). Looking but not seeing: Atypical visual scanning and recognition of faces in 2 and 4-year-old children with autism spectrum disorder. Journal of Autism and Developmental Disorders, 39(12), 1663-1672. doi:10.1007/s10803-009-0803-7

Dawson, G., Webb, S. J., \& McPartland, J. (2005). Understanding the nature of face processing impairment in autism: Insights from behavioral and electrophysiological studies. Developmental Neuropsychology, 27(3), 403-424. doi:10.1207/s15326942dn2703_6

Ellis, H. D. (1990). Developmental trends in face recognition. The Psychologist, 3, 114-119. Falkmer, M., Bjällmark, A., Larsson, M., \& Falkmer, T. (2011). The influences of static and interactive dynamic facial stimuli on visual strategies in persons with Asperger syndrome. Research in Autism Spectrum Disorders, 5(2), 935-940. doi:10.1016/j.rasd.2010.11.003

Falkmer, M., Larsson, M., Bjällmark, A., \& Falkmer, T. (2010). The importance of the eye area in face identification abilities and visual search strategies in persons with Asperger syndrome. Research in Autism Spectrum Disorders, 4(4), 724-730. doi:10.1016/j.rasd.2010.01.011

Falkmer, T., Dahlman, J., Dukic, T., BjäLlmark, A., \& Larsson, M. (2008). Fixation identification in centroid versus start-point modes using eye-tracking data. Perceptual and Motor Skills, 106(3), 710-724. doi:10.2466/pms.106.3.710-724

Gelman, A., \& Hill, J. (2007). Data analysis using regression and multilevel/hierarchical models (Vol. 1). Cambridge University Press New York. Retrieved from http://www.imamu.edu.sa/Scientific_selections/abstracts/Math/Data\%20Analysis\%20Using \%20Regression\%20and\%20MultilevelHierarchical\%20Models.pdf

Happé, F. (1999). Autism: cognitive deficit or cognitive style? Trends in Cognitive Sciences, 3(6), 216-222.

Happé, F., \& Frith, U. (2006). The weak coherence account: detail-focused cognitive style in autism spectrum disorders. Journal of Autism and Developmental Disorders, 36(1), 5-25.

Haxby, J. V., Hoffman, E. A., \& Gobbini, M. I. (2002). Human neural systems for face recognition 
and social communication. Biological Psychiatry, 51(1), 59-67. doi:10.1016/S00063223(01)01330-0

Hernandez, N., Metzger, A., Magné, R., Bonnet-Brilhault, F., Roux, S., Barthelemy, C., \& Martineau, J. (2009). Exploration of core features of a human face by healthy and autistic adults analyzed by visual scanning. Neuropsychologia, 47(4), 1004-1012. doi:10.1016/j.neuropsychologia.2008.10.023

Jemel, B., Mottron, L., \& Dawson, M. (2006). Impaired face processing in autism: Fact or artifact? Journal of Autism and Developmental Disorders, 36(1), 91-106. doi:10.1007/s10803-0050050-5

Karatekin, C. (2007). Eye tracking studies of normative and atypical development. Developmental Review, 27(3), 283-348. doi:10.1016/j.dr.2007.06.006

Kruschke, J. K. (2011). Doing Bayesian data analysis: A tutorial with R and BUGS. Burlington USA: Academic Press Elsevier. Retrieved from http://cognitivesciencesociety.org/uploads/2011-t2.pdf

Kruschke, J. K. (2013). Bayesian estimation supersedes the t Test. Journal of Experimental Psychology: General, 142, 573-603. doi:10.1037/a0029146

Leung, D., Ordqvist, A., Falkmer, T., Parsons, R., \& Falkmer, M. (2013). Facial emotion recognition and visual search strategies of children with high functioning autism and Asperger syndrome. Research in Autism Spectrum Disorders, 7(7), 833-844. doi:10.1016/j.rasd.2013.03.009

Maurer, D., Grand, R. L., \& Mondloch, C. J. (2002). The many faces of configural processing. Trends in Cognitive Sciences, 6(6), 255-260. doi:10.1016/S1364-6613(02)01903-4

McKone, E., Kanwisher, N., \& Duchaine, B. C. (2007). Can generic expertise explain special processing for faces? Trends in Cognitive Sciences, 11(1), 8-15. doi:10.1016/j.tics.2006.11.002

O’Toole, A. J. (2011). Cognitive and computational approaches to face recognition. The Oxford handbook of face perception, 15-30.

Pitcher, D., Charles, L., Devlin, J. T., Walsh, V., \& Duchaine, B. (2009). Triple dissociation of faces, bodies, and objects in extrastriate cortex. Current Biology, 19(4), 319-324. 
doi:10.1016/j.cub.2009.01.007

Plummer, M. (2003). JAGS: A program for analysis of Bayesian graphical models using Gibbs sampling. In Proceedings of the 3rd International Workshop on Distributed Statistical Computing (DSC 2003). March (pp. 20-22). Retrieved from http://www.ci.tuwien.ac.at/Conferences/DSC-2003/Drafts/Plummer.pdf

Plummer, M. (2011). rjags: Bayesian graphical models using MCMC. Retrieved from http://CRAN.R-project.org/package=rjags

R Development Core Team. (2012). R: A language and environment for statistical computing. Vienna, Austria: R Foundation for Statistical Computing. Retrieved from http://www.Rproject.org

Sasson, N. J. (2006). The development of face processing in autism. Journal of Autism and Developmental Disorders, 36(3), 381-394. doi:10.1007/s10803-006-0076-3

Schultz, R. T. (2005). Developmental deficits in social perception in autism: the role of the amygdala and fusiform face area. International Journal of Developmental Neuroscience, 23(2-3), 125-141. doi:10.1016/j.ijdevneu.2004.12.012

Shah, A., \& Frith, U. (1983). An islet of ability in autistic children: A research note. Journal of Child Psychology and Psychiatry, 24(4), 613-620. doi:10.1111/j.1469-7610.1983.tb00137.x

Snow, J., Ingeholm, J. E., Levy, I. F., Caravella, R. A., Case, L. K., Wallace, G. L., \& Martin, A. (2011). Impaired visual scanning and memory for faces in high-functioning autism spectrum disorders: It's not just the eyes. Journal of the International Neuropsychological Society: JINS, 17(6), 1021-1029. doi:10.1017/S1355617711000981

Sterling, L., Dawson, G., Webb, S., Murias, M., Munson, J., Panagiotides, H., \& Aylward, E. (2008). The role of face familiarity in eye tracking of faces by individuals with autism spectrum disorders. Journal of Autism and Developmental Disorders, 38(9), 1666-1675. doi:10.1007/s10803-008-0550-1

Tsao, D. Y., Freiwald, W. A., Tootell, R. B. H., \& Livingstone, M. S. (2006). A cortical region consisting entirely of face-selective cells. Science, 311(5761), 670-674. doi:10.1126/science.1119983

Tsao, D. Y., Moeller, S., \& Freiwald, W. A. (2008). Comparing face patch systems in macaques and 
humans. Proceedings of the National Academy of Sciences, 105(49), 19514-19519. doi:10.1073/pnas.0809662105

Von Hofsten, C., Uhlig, H., Adell, M., \& Kochukhova, O. (2009). How children with autism look at events. Research in Autism Spectrum Disorders, 3(2), 556-569. doi:10.1016/j.rasd.2008.12.003

Weigelt, S., Koldewyn, K., \& Kanwisher, N. (2012). Face identity recognition in autism spectrum disorders: A review of behavioral studies. Neuroscience \& Biobehavioral Reviews, 36(3), 1060-1084. doi:10.1016/j.neubiorev.2011.12.008

Wilmer, J. B., Germine, L., Chabris, C. F., Chatterjee, G., Williams, M., Loken, E., ... Duchaine, B. (2010). Human face recognition ability is specific and highly heritable. Proceedings of the National Academy of Sciences, 107(11), 5238-5241. doi:10.1073/pnas.0913053107

Wilson, C. E., Palermo, R., \& Brock, J. (2012). Visual scan paths and recognition of facial identity in autism spectrum disorder and typical development. PLoS ONE, 7(5), e37681. doi:10.1371/journal.pone.0037681

Yarbus, A. L., Haigh, B., \& Rigss, L. A. (1967). Eye movements and vision (Vol. 2). Plenum press New York. Retrieved from http://library.wur.nl/WebQuery/clc/340878 


\section{Figure Captions}

Figure 1: An example of the presentation of the face identification trial. The puzzle pieces slide in this figure is an example of the eye bisected stimuli.

Figure 2: Left: An example of the puzzle pieces with the eyes whole. From top left in a clockwise direction, the puzzle pieces were classified as 'eye', 'other', 'mouth', 'eye'. 'other' and 'other'. Right: The area labelled as 'A' is classified as the 'eye region' and 'B' as the 'mouth region'.

Figure 3: Fixation odds ratios (Means + 95\% HDIs) for the puzzle pieces. Open circles = controls. Filled circles $=$ ASDs. The left side illustrates the likelihood of fixating on a particular object for controls (open circles) and ASDs (closed circles). The right side presents the contrast in odds ratios between children with ASD and without. Negative contrast deflections indicate less proportional fixations in children with ASD compared to those without.

Figure 4: Fixation odds ratios (Means + 95\% HDIs) for the response slides. Open circles = controls. Filled circles $=$ ASDs. The left side illustrates the likelihood of fixating on a particular object for controls (open circles) and ASDs (closed circles). The right side presents the contrast in odds ratios between children with ASD and without. Negative contrast deflections indicate less proportional fixations in children with ASD compared to those without.

Figure 5: Fixation durations (Means + 95\% HDIs) for the puzzle pieces. Open circles = controls Filled circles $=$ ASDs. Contrast is ASD - controls. The right side presents the contrast in fixation durations between children with and without ASD. Negative contrast deflections indicate less time spent per fixation by children with ASD compared to those without. 
Albrecht et al. 21

Figure 6: Fixation durations (Means + 95\% HDIs) for the response slides. Open circles $=$ controls. Filled circles $=$ ASDs . Contrast is ASD - controls. The right side presents the contrast in fixation durations between children with and without ASD. Negative contrast deflections indicate less time spent per fixation by children with ASD compared to those without. 


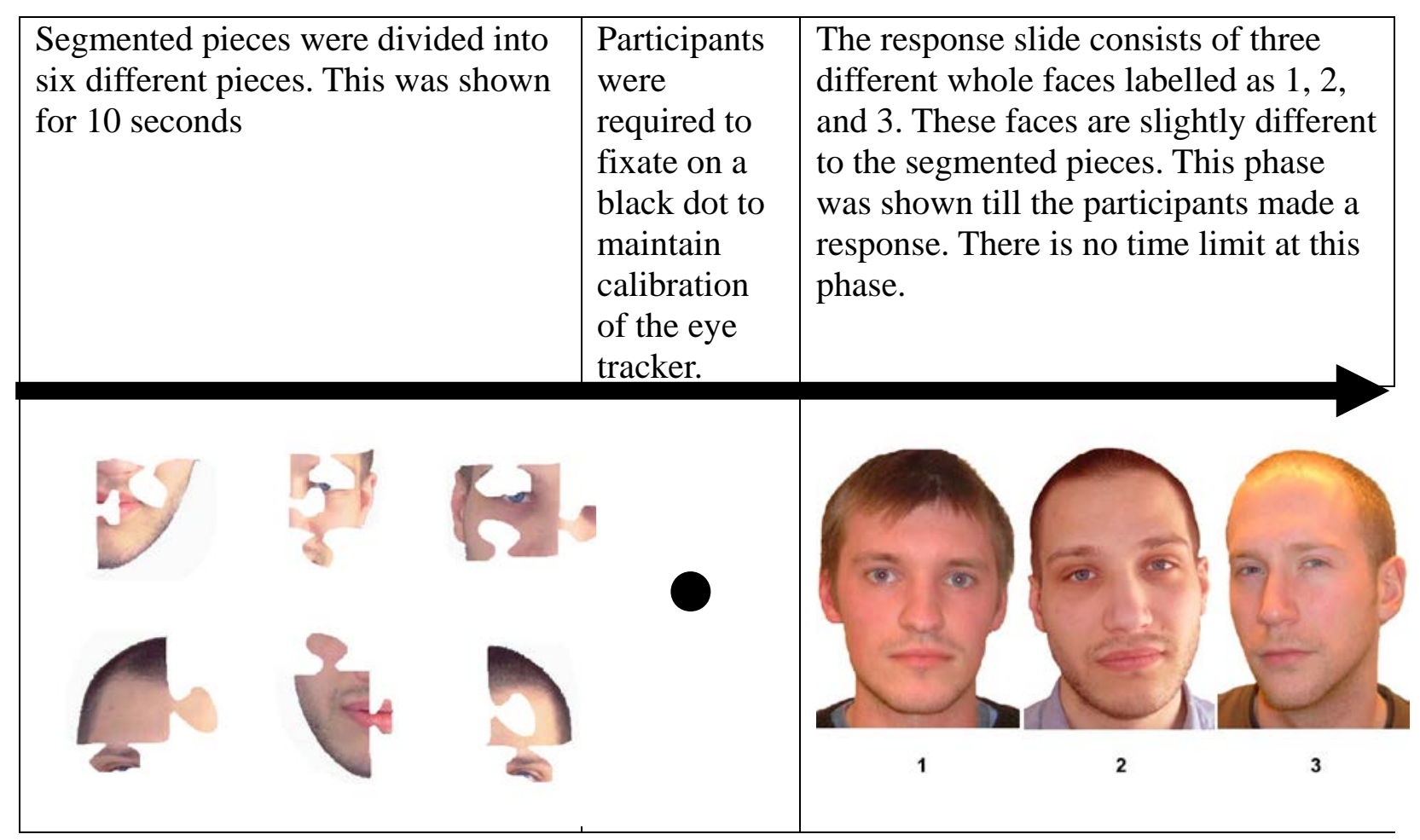

Figure 1: An example of the presentation of the face identification trial. The puzzle pieces slide in this figure is an example of the eye bisected stimuli.
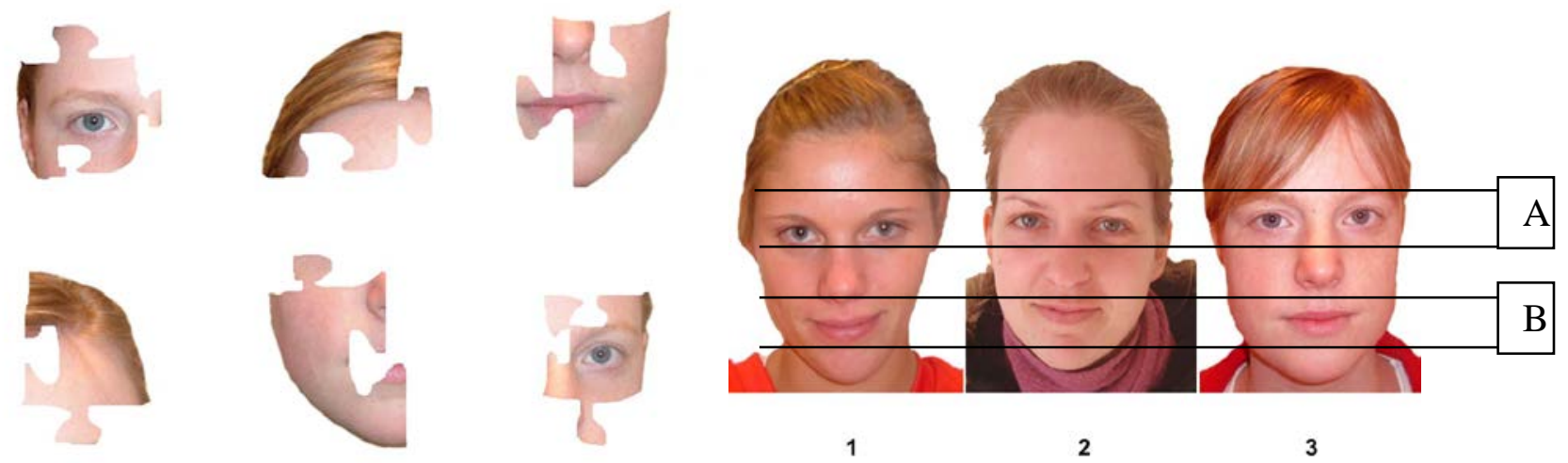

F.igure 2: Left: An example of the puzzle pieces with the eyes whole. From top left in a clockwise direction, the puzzle pieces were classified as 'eye', 'other', 'mouth', 'eye'. 'other' and 'other'. Right: The area labelled as ' $A$ ' is classified as the 'eye region' and ' $\mathrm{B}$ ' as the 'mouth region'. 
- HFA/AS
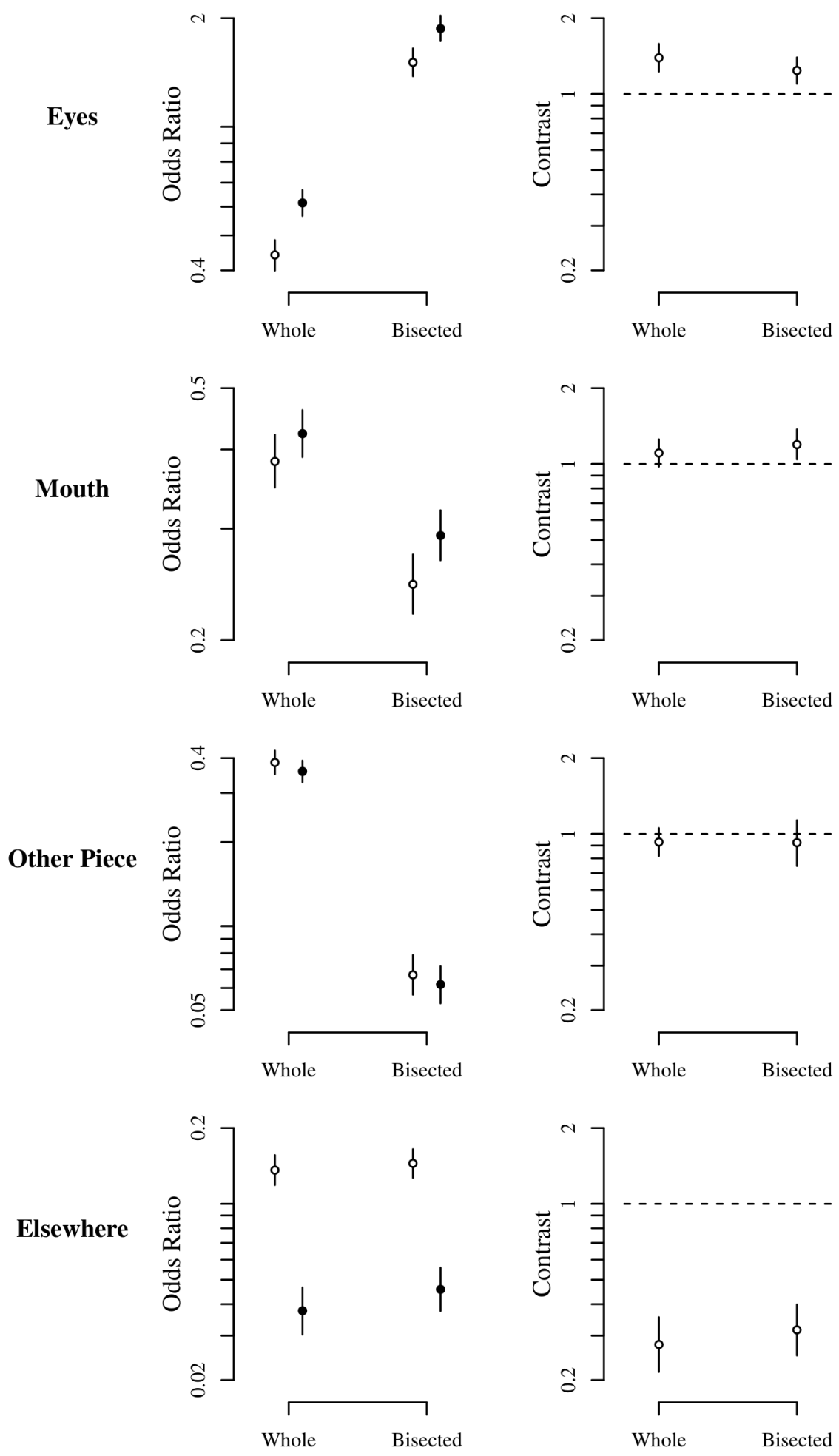

Means (+95\% HDI) Fixation Durations

Contrast HFA/AS-Control 
- Controls

- HFA/AS
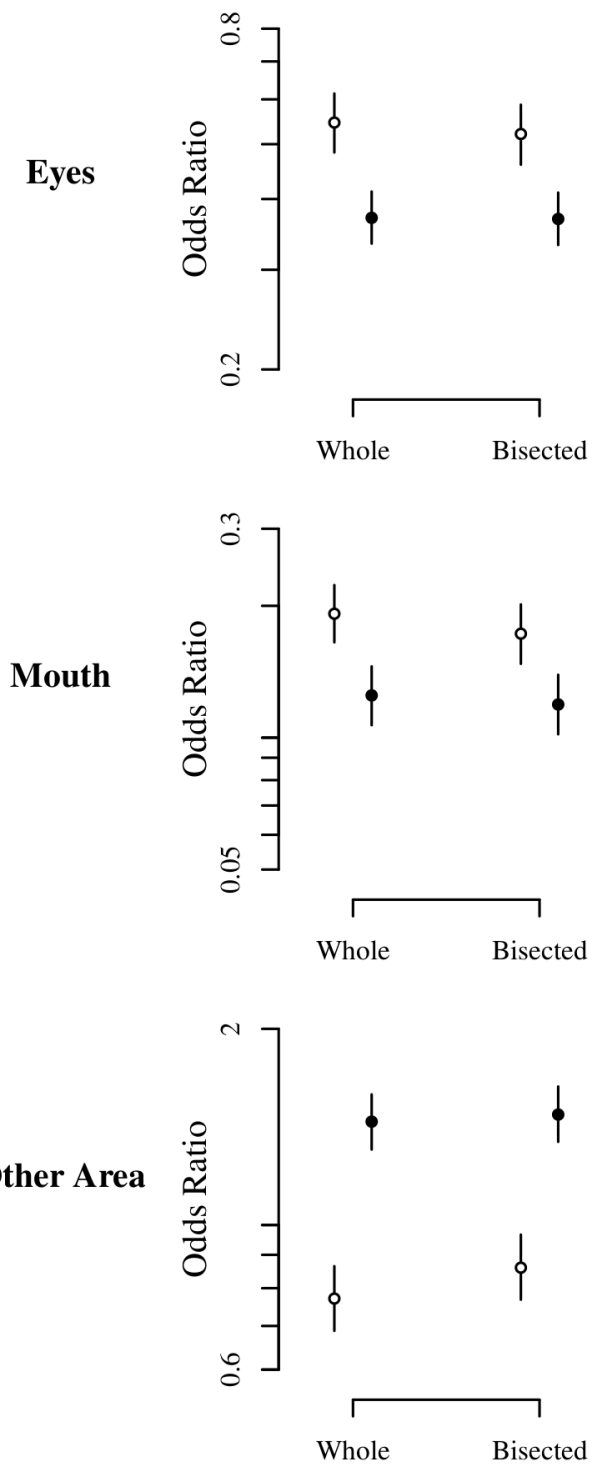

Means (+95\% HDI)

Fixation Durations
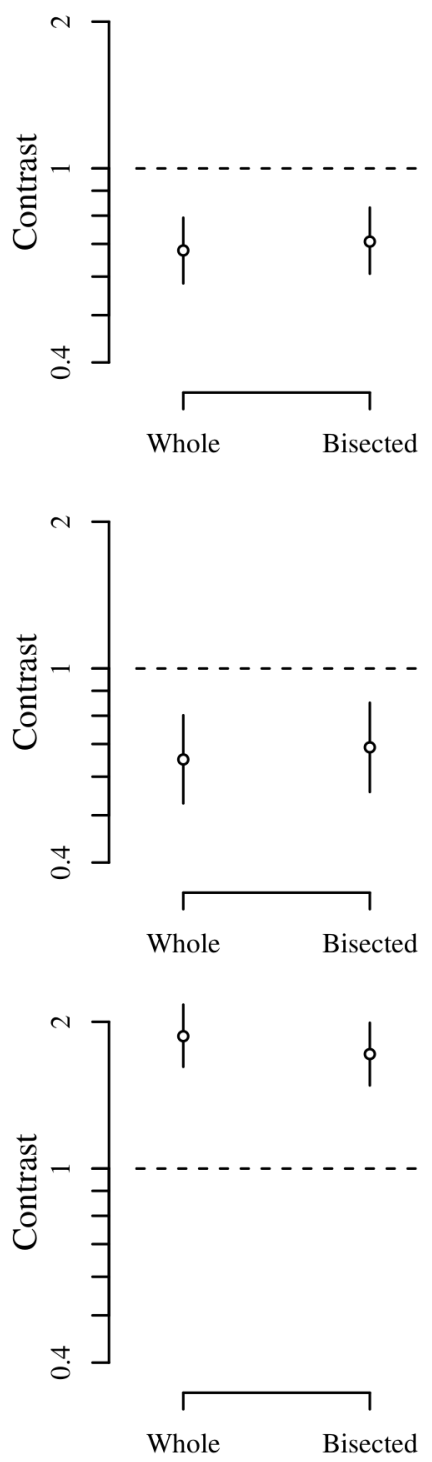

Contrast HFA/AS-Control 
- Controls

- HFA/AS
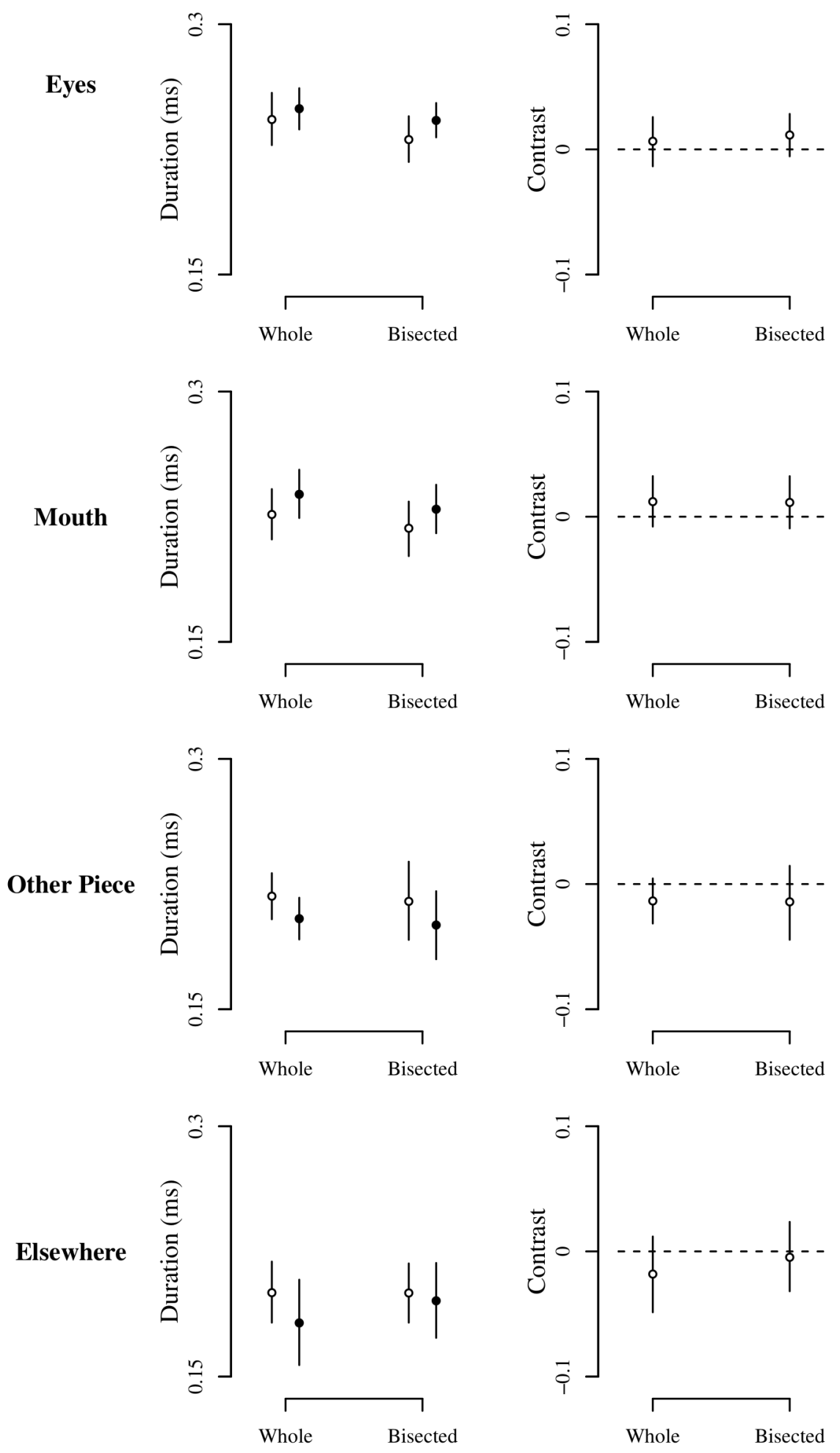

Means (+95\% HDI) Fixation Durations

\author{
Contrast \\ HFA/AS-Control
}



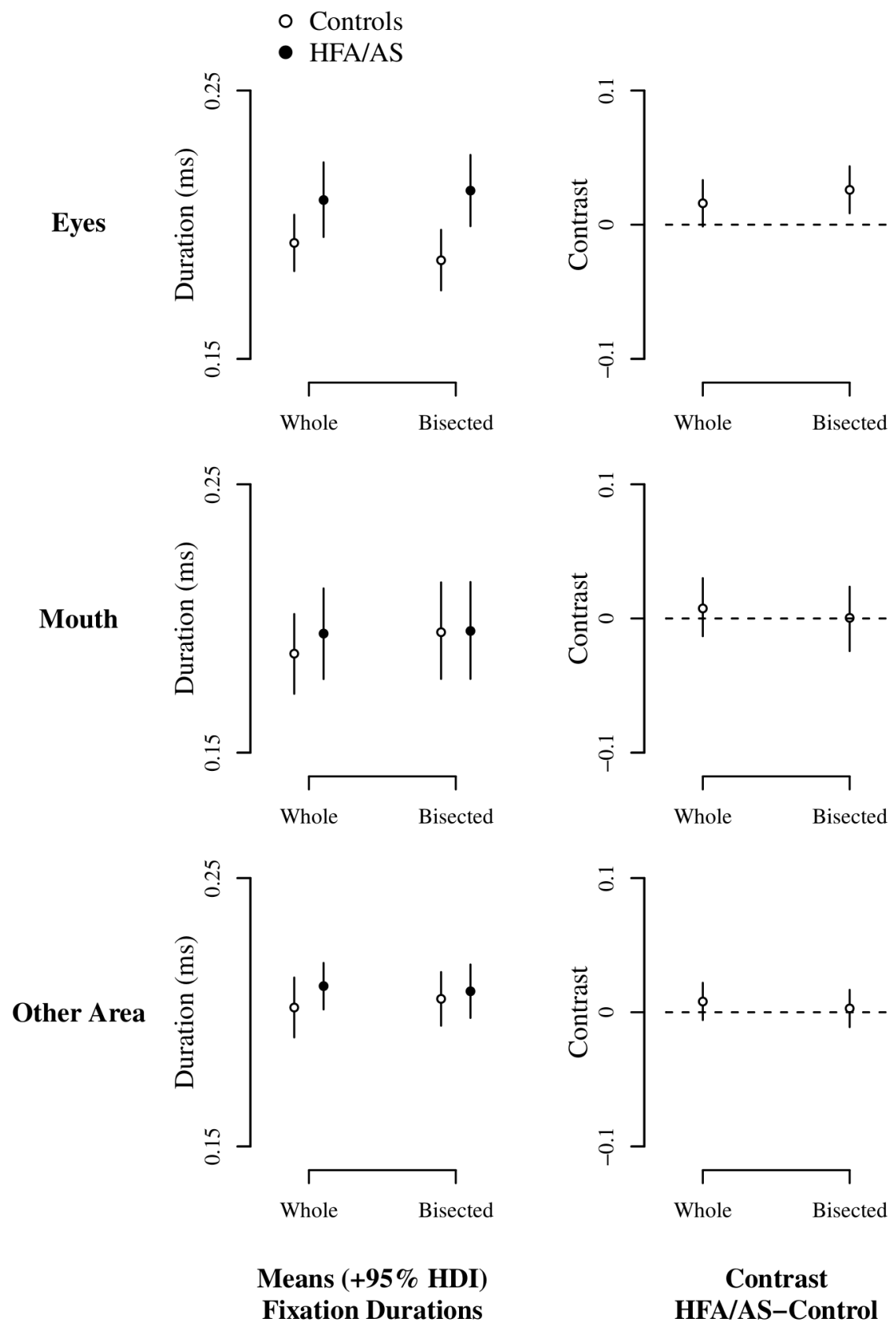

Contrast HFA/AS-Control

\section{Response Slides}

